The Journal of $\mathbf{N}_{\text {onlinear }} \mathbf{S}_{\text {cience and }}$ Applications

http://www.tjnsa.com

\title{
EXISTENCE OF GLOBAL SOLUTIONS FOR IMPULSIVE FUNCTIONAL DIFFERENTIAL EQUATIONS WITH NONLOCAL CONDITIONS
}

\author{
S. SIVASANKARAN ${ }^{1}$, M. MALLIKA ARJUNAN ${ }^{2}$ AND V. VIJAYAKUMAR ${ }^{3, *}$ \\ Dedicated to Professor Themistocles M. Rassias on the occasion of his sixtieth birthday \\ Communicated by Choonkil Park
}

ABstraCt. In this paper, we study the existence of global solutions for a class of impulsive abstract functional differential equation with nonlocal conditions. The results are obtained by using the Leray-Schauder alternative fixed point theorem. An example is provided to illustrate the theory.

\section{INTRODUCTION}

In this work, we discuss the existence of global solutions for an impulsive abstract functional differential equation with nonlocal conditions in the form

$$
\begin{aligned}
u^{\prime}(t) & =A u(t)+f\left(t, u_{t}, u(\rho(t))\right), t \in I=[0, \infty), \\
u_{0} & =g(u, \varphi) \in \mathcal{B}, \\
\Delta u\left(t_{i}\right) & =I_{i}\left(u_{t_{i}}\right),
\end{aligned}
$$

where $A$ is the infinitesimal generator of a $C_{0}$-semigroup of bounded linear operators $(T(t))_{t \geq 0}$ defined on a Banach space $(X,\|\cdot\|) ; I$ is an interval of the form $[0, \infty) ; 0<t_{1}<t_{2}<\cdots<t_{i}<\cdots$ are pre-fixed numbers; the history

$u_{t}:(-\infty, 0] \rightarrow X, u_{t}(\theta)=u(t+\theta)$, belongs to some abstract phase space $\mathcal{B}$ defined axiomatically; $\rho:[0, \infty) \rightarrow[0, \infty), f: I \times \mathcal{B} \times X \rightarrow X, I_{i}: \mathcal{B} \rightarrow X$ are

Date: Received: September 19, 2010; Revised: November 24, 2010.

${ }^{*}$ Corresponding author

(c) 2011 N.A.G.

2000 Mathematics Subject Classification. Primary 34A37, 34K30, 35R10, 35R12, 47D06, $49 \mathrm{~K} 25$.

Key words and phrases. Impulsive functional differential equations; mild solutions; global solutions; semigroup theory. 
appropriate functions and the symbol $\Delta \xi(t)$ represents the jump of the function $\xi$ at $t$, which is defined by $\Delta \xi(t)=\xi\left(t^{+}\right)-\xi\left(t^{-}\right)$.

Differential equations arise in many real world problems such as physics, population dynamics, ecology, biological systems, biotechnology, optimal control, and so forth. Much has been done the assumption that the state variables and systems parameters change continuously. However, one may easily visualize that abrupt changes such as shock, harvesting, and disasters may occur in nature. These phenomena are short-time perturbations whose duration is negligible in comparison with the duration of the whole evolution process. Consequently, it is natural to assume, in modeling these problems, that these perturbations act instantaneously, that is in the form of impulses. The theory of impulsive differential equations has become an active area of investigation due to their applications in the field such as mechanics, electrical engineering, medicine biology and so on; see for instance $[1,3,4,7,8,9,23,26,30,35,37,38]$. For more details on this theory and on its applications we refer to the monographs of Benchohra [10], Lakshmikantham [29], Bainov and Simeonov [5] and Samoilenko and Perestyuk [39], and the papers of $[2,15,16,17,18,25,31]$, where numerous properties of their solutions are studied and detailed bibliographies are given.

The literature concerning differential equations with nonlocal conditions also include ordinary differential equations, partial differential equations, abstract partial functional differential equations. Related to this matter, we cite among others works, $[6,11,12,13,14,20,22,28,33,34]$. Our main results can been seen as a generalization of the work in [21] and the above mentioned impulsive partial functional differential equations.

\section{Preliminaries}

In this paper, $A$ is the infinitesimal generator of a strongly continuous semigroup of bounded linear operators $(T(t))_{t \geq 0}$ defined on a Banach space $(X,\|\cdot\|)$ and $M, \delta$ are positive constants such that $\|T(t)\| \leq M e^{-\delta t}$ for every $t \geq 0$. For the theory of strongly continuous semigroup, refer the reader to Pazy [36].

To consider the impulsive condition (1.3), it is convenient to introduce some additional concepts and notations. We say that a function $u:[\sigma, \tau] \rightarrow X$ is a normalized piecewise continuous function on $[\sigma, \tau]$ if $u$ is piecewise continuous and left continuous on $(\sigma, \tau]$. We denote by $\mathcal{P C}([\sigma, \tau] ; X)$ the space formed by all normalized piecewise continuous functions from $[\sigma, \tau]$ into $X$. In particular, we introduce the space $\mathcal{P C}$ formed by all functions $u:[0, a] \rightarrow X$ such that $u$ is continuous at $t \neq t_{i}, i=1, \ldots, n$. It is clear that, $\mathcal{P C}([\sigma, \tau] ; X)$ endowed with the norm of uniform convergence, is a Banach space. On the other hand, the notation $\mathcal{P C}([0, \infty) ; X)$ stands for the space formed by all bounded normalized piecewise continuous functions $u:[0, \infty) \rightarrow X$ such that $\left.u\right|_{[0, a]} \in \mathcal{P C}([0, a] ; X)$ for all $a>0$, endowed with the uniform convergence topology.

In what follows, for the case $I=[0, a]$, we set $t_{0}=0, t_{n+1}=a$, and for $u \in \mathcal{P C}([0, a] ; X)$, we denote by $\widetilde{u}_{i} \in C\left(\left[t_{i}, t_{i+1}\right] ; X\right), i=0,1, \ldots, n$, the function 
given by

$$
\widetilde{u}_{i}(t)= \begin{cases}u(t), & \text { for } t \in\left(t, t_{i+1}\right], \\ u\left(t_{i}^{+}\right), & \text {for } t=t_{i} .\end{cases}
$$

Moreover, for $\mathcal{B} \subset \mathcal{P C}$, we employ the notation $\widetilde{B}_{i}, i=0,1, \ldots, n$, for the sets $\widetilde{B}_{i}=\left\{\widetilde{u}_{i}: u \in B\right\}$.

Lemma 2.1. A set $\mathcal{B} \subset \mathcal{P C}$ is relatively compact in $\mathcal{P C}([0, a] ; X)$ if, and only if, the set $\widetilde{B}_{i}$, is relatively compact in the space $C\left(\left[t_{i}, t_{i+1}\right] ; X\right)$ for every $i=0,1, \ldots, n$.

In this paper, the phase space $\mathcal{B}$ is a linear space of functions mapping $(-\infty, 0]$ into $X$ endowed with a seminorm $\|\cdot\|_{\mathcal{B}}$ and verifying the following axioms.

(A) If $x:(-\infty, a) \rightarrow X, a>0$, is continuous on $[0, a)$ and $x_{0} \in \mathcal{B}$, then for every $t \in[0, a)$ the following conditions hold:

(i) $x_{t}$ is in $\mathcal{B}$.

(ii) $\|x(t)\| \leq H\left\|x_{t}\right\|_{\mathcal{B}}$.

(iii) $\left\|x_{t}\right\|_{\mathcal{B}} \leq K(t) \sup \{\|x(s)\|: 0 \leq s \leq t\}+M(t)\left\|x_{0}\right\|_{\mathcal{B}}$, where $H>0$ is a constant; $K, M:[0, \infty) \rightarrow[1, \infty), K$ is continuous, $M$ is locally bounded and $H, K, M$ are independent of $x(\cdot)$.

(B) The space $\mathcal{B}$ is complete.

Remark 2.2. In this paper, $\mathcal{K}$ is a positive constant such that $\sup _{t \geq 0}\{K(t), M(t)\}$ $\leq \mathcal{K}$. We know from that the functions $M(\cdot), K(\cdot)$ are bounded if, for instance, $\mathcal{B}$ is a fading memory space, see [27, pp. 190] for additional details.

Example 2.3. The phase space $\mathcal{P} \mathcal{C}_{r} \times L^{p}(\rho, X)$

Let $r \geq 0,1 \leq p<\infty$, and $\rho:(-\infty,-r] \rightarrow \mathbb{R}$ be a non-negative measurable function which satisfies the conditions (g-5)-(g-6) of [14]. Briefly, this means that $\rho$ is locally integrable and there exists a non-negative locally bounded function $\gamma$ on $(-\infty, 0]$ such that $\rho(\xi+\theta) \leq \gamma(\xi) \rho(\theta)$, for all $\xi \leq 0$ and $\theta \in(-\infty,-r) \backslash N_{\xi}$, where $N_{\xi} \subseteq(-\infty,-r)$ is a set whose Lebesgue measure zero.

The space $\mathcal{B}=\mathcal{P C}_{r} \times L^{p}(\rho, X)$ consists of all classes of functions $\psi:(-\infty, 0] \rightarrow$ $X$ such that $\psi$ is continuous on $[-r, 0]$, Lebesgue-measurable, and $\rho\|\psi\|^{p}$ is Lebesgue integrable on $(-\infty,-r)$. The seminorm in $\mathcal{P C}_{r} \times L^{p}(\rho, X)$ is defined as follows:

$$
\|\psi\|_{\mathcal{B}}=\sup \{\|\psi(\theta)\|:-r \leq \theta \leq 0\}+\left(\int_{-\infty}^{-r} \rho(\theta)\|\psi(\theta)\|^{p} d \theta\right)^{\frac{1}{p}}
$$

The space $\mathcal{B}=\mathcal{P C}_{r} \times L^{p}(\rho, X)$ satisfies axioms $(\mathbf{A})$ and $(\mathbf{B})$. Moreover, when $r=0$ and $p=2$, one can take $H=1, M(t)=\gamma(-t)^{\frac{1}{2}}$ and $K(t)=1+$ $\left(\int_{-t}^{0} \rho(\theta) d \theta\right)^{\frac{1}{2}}$ for $t \geq 0$ (see [27, Theorem 1.3.8]). We also note that if the conditions (g-5)-(g-7) of [27] hold, then $\mathcal{B}$ is a uniform fading memory.

For additional details concerning phase space, we cite [27]

In this paper, $h:[0, \infty) \rightarrow \mathbb{R}$ be a positive, continuous and non-decreasing function such that $h(0)=1$ and $\lim _{t \rightarrow \infty} h(t)=\infty$. In the sequel, $\mathcal{P C}([0, \infty) ; X)$ 
and $\left.(\mathcal{P C})_{h}\right)^{0}(X)$ are the spaces defined by

$$
\begin{gathered}
\mathcal{P C}([0, \infty) ; X)=\left\{\begin{array}{l}
x_{\left.\right|_{[0, a]} \in \mathcal{P C}([0, a] ; X),} \quad \forall a \in(0,+\infty) \backslash\left\{t_{i}: i \in \mathbb{N}\right\}, \\
x:[0, \infty) \rightarrow X: \begin{array}{l}
x_{0}=0, \\
\|x\|_{\infty}=\sup _{t \geq 0}\|x(t)\|<+\infty,
\end{array} \\
(\mathcal{P C})_{h}^{0}(X)=\left\{x \in \mathcal{P C}([0, \infty) ; X): \lim _{t \rightarrow \infty} \frac{\|x(t)\|}{h(t)}=0\right\},
\end{array}\right.
\end{gathered}
$$

endowed with the norms $\|x\|_{\infty}=\sup _{t \geq 0}\|x(t)\|$ and $\|x\|_{\mathcal{P C}_{h}}=\sup _{t \geq 0} \frac{\|x(t)\|}{h(t)}$, respectively.

Additionally, we define the space

$$
\mathcal{B P C}_{h}^{0}(X)=\left\{u \in \mathcal{P C}(\mathbb{R}, X): u_{0} \in \mathcal{B}, u_{\left.\right|_{[0, \infty)}} \in \mathcal{P C}_{h}^{0}(X)\right\}
$$

endowed with the norm $\|u\|_{\mathcal{B P C}_{h}^{0}}=\left\|u_{0}\right\|_{\mathcal{B}}+\left\|u_{[0, \infty)}\right\|_{h}$.

We recall here the following compactness criterion.

Lemma 2.4. A bounded set $\mathcal{B} \subset(\mathcal{P C})_{h}^{0}(X)$ is relatively compact in $(\mathcal{P C})_{h}^{0}(X)$ if, and only if:

(a) The set $B_{a}=\left\{u_{\left.\right|_{[0, a]}}: u \in B\right\}$ is relatively compact in $\mathcal{P C}([0, a] ; X)$, for all $a \in(0, \infty) \backslash\left\{t_{i} ; i \in \mathbb{N}\right\}$.

(b) $\lim _{t \rightarrow \infty} \frac{\|x(t)\|}{h(t)}=0$, uniformly for $x \in B$.

Theorem 2.5. ([19, Theorem 6.5.4] Leray-Schauder Alternative Theorem). Let $D$ be a closed convex subset of a Banach space $\left(Z,\|\cdot\|_{Z}\right)$ and assume that $0 \in D$. Let $F: D \rightarrow D$ be a completely continuous map, then the set $\{x \in D: x=\lambda F(x)$, $0<\lambda<1\}$ is unbounded or the map $F$ has a fixed point in $D$.

Theorem 2.6. ([32, Theorem 4.3.2]). Let $D$ be a convex, bounded and closed subset of a Banach space $\left(Z,\|\cdot\|_{Z}\right)$ and $F: D \rightarrow D$ be a condensing map. Then $F$ has a fixed point in $D$.

\section{Existence of MiLd solutions}

In this section, we study the existence of mild solutions for the nonlocal abstract Cauchy problem (1.1)-(1.3). To treat this system, we introduce the following conditions.

$\mathbf{H}_{1}$ The function $\rho:[0, \infty) \rightarrow[0, \infty)$ is continuous and $\rho(t) \leq t$ for every $t \geq 0$.

$\mathbf{H}_{2}$ The function $f: I \times \mathcal{B} \times X \rightarrow X$ is continuous and there exist an integrable function $m:[0, \infty) \rightarrow[0, \infty)$ and a nondecreasing continuous function $W:[0, \infty) \rightarrow(0, \infty)$ such that $\|f(t, \psi, x)\| \leq m(t) W\left(\|\psi\|_{\mathcal{B}}+\|x\|\right)$, for every $(t, \psi, x) \in[0, \infty) \times \mathcal{B} \times X$.

$\mathbf{H}_{3}$ The function $g: \mathcal{B P} \mathcal{C}_{h}^{0}(X) \times \mathcal{B} \rightarrow \mathcal{B}$ is continuous and there exists $L_{g} \geq 0$ such that

$$
\|g(u, \varphi)-g(v, \varphi)\|_{\mathcal{B}} \leq L_{g}\|u-v\|_{\mathcal{B P C}_{h}^{0}}, \quad u, v \in \mathcal{B P}_{h}^{0}(X)
$$


$\mathbf{H}_{4}$ The function $g(\cdot, \varphi): \mathcal{B P C}_{h}^{0}(X) \rightarrow \mathcal{B}$ is completely continuous and uniformly bounded. In the sequel, $N$ is a positive constant such that $\|g(\psi, \varphi)\|_{\mathcal{B}} \leq N$ for every $\psi \in \mathcal{B P C}_{h}^{0}(X)$.

$\mathbf{H}_{5}$ The maps $I_{i}: \mathcal{B} \rightarrow X$ are completely continuous and uniformly bounded, $i \in \mathbb{N}$. In what follows, we use the notation $N_{i}=\sup \left\{\left\|I_{i}(\phi)\right\|: \phi \in \mathcal{B}\right\}$.

$\mathbf{H}_{6}$ There are positive constants $L_{i}$, such that

$$
\left\|I_{i}\left(\psi_{1}\right)-I_{i}\left(\psi_{2}\right)\right\| \leq L_{i}\left\|\psi_{1}-\psi_{2}\right\|_{\mathcal{B}}, \psi_{1}, \psi_{2} \in \mathcal{B}, i \in \mathbb{N} .
$$

In this work, we adopt the following concept of mild solutions for (1.1)-(1.3).

Definition 3.1. A function $u \in \mathcal{P C}(\mathbb{R}, X)$ is called a mild solution of system (1.1)-(1.3) if $u_{0}=g(u, \varphi)$ and

$$
\begin{aligned}
u(t)=T(t) g(u, \varphi)(0) & +\int_{0}^{t} T(t-s) f\left(s, u_{s}, u(\rho(s))\right) d s \\
& +\sum_{0<t_{i}<t} T\left(t-t_{i}\right) I_{i}\left(u_{t_{i}}\right), t \in I=[0, \infty) .
\end{aligned}
$$

Now, we can establish the principal results of this section.

Theorem 3.2. Assume $\mathbf{H}_{1}, \mathbf{H}_{2}, \mathbf{H}_{4}, \mathbf{H}_{5}$ and $\mathbf{H}_{6}$ be hold and that the following properties are verified.

(a) The set $\left\{T(t) f(s, \psi, x):(s, \psi, x) \in[0, t] \times B_{r}(0, \mathcal{B}) \times B_{r}(0, X)\right\}$ is relatively compact in $X$ for every $t \geq 0$ and all $r>0$.

(b) For every $K>0, \lim _{t \rightarrow \infty} \frac{1}{h(t)} \int_{0}^{t} m(s)(K h(s)) d s=0$.

If $M \mathcal{K}(1+H) \int_{0}^{\infty} m(s) d s<\int_{c}^{\infty} \frac{d s}{W(s)}$, where $c=\mathcal{K} N(1+H)(1+M H)$ $+M \mathcal{K} \sum_{i=1}^{\infty} N_{i}$, then there exists a mild solution for the system (1.1)-(1.3).

Proof. Let $\Gamma: \mathcal{B P C}_{h}^{0}(I: X) \rightarrow \mathcal{B P C}_{h}^{0}(X)$ be the operator defined by $(\Gamma u)_{0}=$ $g(u, \varphi)$ and

$$
\begin{aligned}
\Gamma u(t)=T(t) g(u, \varphi)(0)+\int_{0}^{t} T & (t-s) f\left(s, u_{s}, u(\rho(s))\right) d s \\
& +\sum_{0<t_{i}<t} T\left(t-t_{i}\right) I_{i}\left(u_{t_{i}}\right), t \geq 0 .
\end{aligned}
$$

Next we prove that $\Gamma$ verifies the conditions in Theorem 2.5. To begin, we note that for $u \in \mathcal{B P C}_{h}^{0}(X)$ and $t \geq 0,\|u(t)\| \leq\left\|u_{I}\right\|_{h} h(t)$. If $\mathcal{K}$ is the constant in Remark 2.2, then

$$
\frac{\|\Gamma u(t)\|}{h(t)} \leq \frac{M N H}{h(t)}+\frac{M}{h(t)} \int_{0}^{t} m(s) W\left(2 \mathcal{K}\|u\|_{\mathcal{B P C}_{h}^{0}} h(s)\right) d s+\frac{M}{h(t)} \sum_{i=1}^{\infty} N_{i},
$$

which from (b) permits us to conclude that $\Gamma$ is a function from $\mathcal{B P C}_{h}^{0}(X)$ into $\mathcal{B P C}_{h}^{0}(X)$.

Let $\left(x^{n}\right)_{n \in \mathbb{N}}$ be a sequence in $\mathcal{B P C}_{h}^{0}(X)$ and $x \in \mathcal{B P C}_{h}^{0}(X)$ such that $x^{n} \rightarrow x$ in $\mathcal{B P C}_{h}^{0}(X)$. Let $\varepsilon>0$ and $\eta=\sup _{n \in \mathbb{N}}\left\|x^{n}\right\|_{\mathcal{B P C}_{h}^{0}}$. From condition (b), we can 
select $L>0$ such that

$$
\frac{2 M N H}{h(t)}+\frac{M}{h(t)} \int_{0}^{t} m(s) W(2 \mathcal{K} \eta h(s)) d s+\frac{2 M}{h(t)} \sum_{i=1}^{\infty} N_{i} \leq \frac{\varepsilon}{2}, \quad t \geq L
$$

From the properties of the functions $f, g$, we can choose $N_{\varepsilon} \in \mathbb{N}$ such that

$$
\begin{array}{r}
\left\|g\left(x^{n}, \varphi\right)-g(x, \varphi)\right\|_{\mathcal{B}} \leq \frac{\varepsilon}{6(M H+1)}, \\
\int_{0}^{L}\left\|f\left(s, x_{s}^{n}, x^{n}(\rho(s))\right)-f\left(s, x_{s}, x(\rho(s))\right)\right\| d s \leq \frac{\varepsilon}{6 M},
\end{array}
$$

and

$$
\sum_{0 \leq t_{i} \leq L}\left\|I_{i}\left(x_{t_{i}}^{n}\right)-I_{i}\left(x_{t_{i}}\right)\right\| \leq \frac{\varepsilon}{6 M}, \quad n \geq N_{\varepsilon}
$$

for every $n \geq N_{\varepsilon}$. Under these conditions, we see that

$$
\sup \left\{\frac{\left\|\Gamma x^{n}(t)-\Gamma x(t)\right\|}{h(t)}: t \in[0, L], n \geq N_{\varepsilon}\right\} \leq \frac{\varepsilon}{2} .
$$

Moreover, for $t \geq L$ and $n \geq N_{\varepsilon}$, we find that

$$
\begin{aligned}
\frac{\left\|\Gamma x^{n}(t)-\Gamma x(t)\right\|}{h(t)} \leq & M \frac{\left\|g\left(x^{n}, \varphi\right)(0)-g(x, \varphi)(0)\right\|}{h(t)}+\frac{2 M}{h(t)} \sum_{i=1}^{\infty} N_{i} \\
& +\frac{M}{h(t)} \int_{0}^{t}\left\|f\left(s, x_{s}^{n}, x^{n}(\rho(s))\right)-f\left(s, x_{s}, x(\rho(s))\right)\right\| d s, \\
\leq & \frac{2 M N H}{h(t)}+\frac{M}{h(t)} \int_{0}^{t} m(s) W(2 \mathcal{K} \eta h(s)) d s+\frac{2 M}{h(t)} \sum_{i=1}^{\infty} N_{i},
\end{aligned}
$$

and hence,

$$
\sup \left\{\frac{\left\|\Gamma x^{n}(t)-\Gamma x(t)\right\|}{h(t)}: t \geq L, n \geq N_{\varepsilon}\right\} \leq \frac{\varepsilon}{2} .
$$

From the inequalities (3.2)-(3.6), it follows that $\left\|\Gamma x^{n}-\Gamma x\right\|_{\mathcal{B P C}_{h}^{0}} \leq \varepsilon$ for every $n \geq N_{\varepsilon}$, which proves that $\Gamma$ is continuous.

To prove that $\Gamma$ is completely continuous, we introduce the decomposition $\Gamma=\Gamma_{1}+\Gamma_{2}+\Gamma_{3}$, where $\left(\Gamma_{1} u\right)_{0}=g(u, \varphi),\left(\Gamma_{2} u\right)_{0}=0,\left(\Gamma_{3} u\right)_{0}=0$ and

$$
\begin{aligned}
& \Gamma_{1} u(t)=T(t) g(u, \varphi)(0), t \geq 0 \\
& \Gamma_{2} u(t)=\int_{0}^{t} T(t-s) f\left(s, u_{s}, u(\rho(s))\right) d s, t \geq 0 \\
& \Gamma_{3} u(t)=\sum_{0<t_{i}<t} T\left(t-t_{i}\right) I_{i}\left(u_{t_{i}}\right), t \geq 0
\end{aligned}
$$

From Lemma 2.4 and the properties of the semigroup $(T(t))_{t \geq 0}$, it is easy to see that $\Gamma_{1}$ is completely continuous. Next, we prove that $\Gamma_{2}$ is also completely continuous. From [24, Lemma 3.1], we infer that, $\left.\Gamma_{2} B_{r}\left(0, \mathcal{B P C}_{h}^{0}\right)\right|_{[0, a]}=\left\{\left.\Gamma_{2} x\right|_{[0, a]}\right.$ : 
$\left.x \in B_{r}\left(0, \mathcal{B P C}_{h}^{0}\right)\right\}$ is relatively compact in $\mathcal{P C}([0, a] ; X)$ for every $a>0$. Considering this property, Lemma 2.4 and the fact that

$$
\frac{\left\|\Gamma_{2} x(t)\right\|}{h(t)} \leq \frac{M}{h(t)} \int_{0}^{t} m(s) W(2 \mathcal{K} r h(s)) d s \rightarrow 0, \text { as } t \rightarrow \infty
$$

uniformly for $x \in B_{r}\left(0, \mathcal{B P C}_{h}^{0}\right)$, we can conclude that the set $\Gamma_{2}\left(B_{r}\left(0, \mathcal{B P C}_{h}^{0}\right)\right)$ is relatively compact in $\mathcal{B P C}_{h}^{0}(X)$ for every $r>0$. Next, by using Lemma 2.1 , we prove that $\Gamma_{3}$ is also completely continuous. We observe that the continuity of $\Gamma_{3}$ is obvious. On the other hand, for $r>0, t \in\left[t_{i}, t_{i+1}\right], i \geq 1$, and $u \in B_{r}=$ $B_{r}(0, \mathcal{P C}([0, a] ; X))$, we have that there exists $\widetilde{r}>0$ such that

$$
\left[\widetilde{\Gamma_{3} u}\right]_{i}(t) \in \begin{cases}\sum_{j=1}^{i} T\left(t-t_{j}\right) I_{j}\left(B_{\widetilde{r}}(0 ; \mathcal{B})\right), & t \in\left(t_{i}, t_{i+1}\right) \\ \sum_{j=1}^{i} T\left(t_{i+1}-t_{j}\right) I_{j}\left(B_{\widetilde{r}}(0 ; \mathcal{B})\right), & t=t_{i+1} \\ \sum_{j=1}^{i-1} T\left(t_{i}-t_{j}\right) I_{j}\left(B_{\widetilde{r}}(0 ; \mathcal{B})\right)+I_{i}\left(B_{\widetilde{r}}(0 ; \mathcal{B})\right), & t=t_{i}\end{cases}
$$

where $B_{\widetilde{r}}(0 ; \mathcal{B})$ is an open ball of radius $\widetilde{r}$. From condition $\mathbf{H}_{\mathbf{5}}$ it follows that $\left[\widehat{\Gamma_{3}\left(B_{r}\right)}\right]_{i}(t)$ is relatively compact in $X$, for all $t \in\left[t_{i}, t_{i+1}\right], i \geq 1$. Moreover, using the compactness of the operators $\left\{I_{i}\right\}_{i}$ and the continuity of $(T(t))_{t \geq 0}$, we conclude that for $\varepsilon>0$, there exists $\delta>0$ such that

$$
\|T(t) z-T(s) z\| \leq \varepsilon, \quad z \in \bigcup_{i=1}^{n} I_{i}\left(B_{\widetilde{r}}(0, \mathcal{B})\right)
$$

for all $t_{i}, i=1, \cdots, n, t, s \in\left(t_{i}, t_{i+1}\right]$ with $|t-s|<\delta$. Under these conditions, for $u \in B_{r}, t \in\left[t_{i}, t_{i+1}\right], i \geq 0$, and $0<|h|<\delta$ with $t+h \in\left[t_{i}, t_{i+1}\right]$, we have that

$$
\left.\| \widetilde{\Gamma_{3} u}\right]_{i}(t+h)-\left[\widetilde{\Gamma_{3} u}\right]_{i}(t) \| \leq M n \varepsilon, \quad t \in I,
$$

which proves that the set of functions $\left[\widetilde{\Gamma_{3}\left(B_{r}\right)}\right]_{i}, i \geq 0$, is uniformly equicontinuous. Now, from Lemma 2.1, we conclude that $\Gamma_{3}$ is completely continuous. Thus, $\Gamma$ is completely continuous.

To finish the proof, we obtain a priori estimates for the solutions of the integral equation $\{u=\lambda \Gamma u, \lambda \in(0,1)\}$. Let $\lambda \in(0,1)$ and $u^{\lambda} \in \mathcal{B P C}_{h}^{0}(X)$ be a solution of $\lambda \Gamma z=z$. By using the notation $\alpha^{\lambda}(t)=\mathcal{K}\left(\sup _{s \in[0, t]}\left\|u^{\lambda}(s)\right\|+\left\|u_{0}^{\lambda}\right\|_{\mathcal{B}}\right)$, for $t \in I$ we see that

$$
\begin{aligned}
\left\|u^{\lambda}(t)\right\| & \leq M N H+M \int_{0}^{t} m(s) W\left(\left\|u_{s}^{\lambda}\right\|_{\mathcal{B}}+\left\|u^{\lambda}(\rho(s))\right\|\right) d s+M \sum_{i=1}^{\infty} N_{i}, \\
& \leq M N H+M \int_{0}^{t} m(s) W\left((1+H) \alpha^{\lambda}(s)\right) d s+M \sum_{i=1}^{\infty} N_{i},
\end{aligned}
$$


and hence,

$$
\begin{aligned}
& \alpha^{\lambda}(t) \\
& \quad \leq \mathcal{K}\|g(u, \varphi)\|_{\mathcal{B}}+\mathcal{K} M N H+\mathcal{K} M \int_{0}^{t} m(s)\left(W(1+H) \alpha^{\lambda}(s)\right) d s+\mathcal{K} M \sum_{i=1}^{\infty} N_{i}, \\
& \quad \leq \mathcal{K} N(1+M H)+\mathcal{K} M \int_{0}^{t} m(s) W\left((1+H) \alpha^{\lambda}(s)\right) d s+\mathcal{K} M \sum_{i=1}^{\infty} N_{i} .
\end{aligned}
$$

Denoting by $\beta_{\lambda}$ the right hand side of last inequality, we see that

$$
\beta_{\lambda}^{\prime}(t) \leq \mathcal{K} M m(t) W\left((1+H) \beta_{\lambda}(t)\right)
$$

which implies that

$$
\int_{(1+H) \beta_{\lambda}(0)=c}^{(1+H) \beta_{\lambda}(t)} \frac{d s}{W(s)} \leq M \mathcal{K}(1+H) \int_{0}^{\infty} m(s) d s<\int_{c}^{\infty} \frac{d s}{W(s)}
$$

This inequality permits us to conclude that the set of functions $\left\{\beta_{\lambda}: \lambda \in(0,1)\right\}$ is bounded in $C(\mathbb{R})$ and as consequence that the set $\left\{u^{\lambda} ; \lambda \in(0,1)\right\}$ is bounded in $\mathcal{B P C}_{h}^{0}(X)$.

Now the assertion is a consequence of Theorem 2.5. The proof is complete.

Theorem 3.3. Assume assumptions $\mathbf{H}_{\mathbf{1}}, \mathbf{H}_{\mathbf{2}}, \mathbf{H}_{\mathbf{3}}, \mathbf{H}_{\mathbf{5}}$ and $\mathbf{H}_{\mathbf{6}}$ be hold. If the conditions $(\mathbf{a})$ and $(\mathbf{b})$ of Theorem 3.2 are valid and

$$
L_{g}(1+M H)+M \lim _{r \rightarrow \infty} \inf \int_{0}^{+\infty} \frac{m(s) W((1+2 \mathcal{K}) r h(s))}{r h(s)} d s+M \sum_{i=1}^{\infty} L_{i}<1
$$

then there exists a mild solution of (1.1)-(1.3).

Proof. Let $\Gamma, \Gamma_{1}, \Gamma_{2}, \Gamma_{3}$ be the operators introduced in the proof of Theorem 3.2, we claim that there exists $r>0$ such that $\Gamma\left(B_{r}\right) \subset B_{r}$, where $B_{r}=$ $B_{r}\left(0, \mathcal{B P C}_{h}^{0}(X)\right)$. In fact, if this property is false, then for every $r>0$, there 
exist $x^{r} \in B_{r}$ and $t^{r} \geq 0$ such that $r<\left\|\left(\Gamma x^{r}\right)_{0}\right\|_{\mathcal{B}}+\left\|\frac{\Gamma x^{r}\left(t^{r}\right)}{h\left(t^{r}\right)}\right\|$. Consequently

$$
\begin{aligned}
r \leq & \left\|g\left(x^{r}, \varphi\right)\right\|_{\mathcal{B}}+M \frac{\left\|g\left(x^{r}, \varphi\right)(0)\right\|}{h\left(t^{r}\right)} \\
& +\frac{M}{h\left(t^{r}\right)} \int_{0}^{t^{r}} m(s) W\left(\mathcal{K}\left\|x_{0}^{r}\right\|_{\mathcal{B}}+\mathcal{K} \sup _{\theta \in[0, s]}\left\|x^{r}(\theta)\right\|+\left\|x^{r}(\rho(s))\right\|\right) d s \\
& +\frac{M}{h\left(t^{r}\right)} \sum_{i=1}^{\infty}\left\|I_{i}(0)\right\|+M \sum_{i=1}^{\infty} \frac{L_{i}\left\|x_{t_{i}}\right\|}{h\left(t^{r}\right)} \\
\leq & L_{g}\left\|x^{r}\right\|_{\mathcal{B} \mathcal{P} C_{h}^{o}}+\|g(0, \varphi)\|_{\mathcal{B}}+\frac{M H}{h\left(t^{r}\right)} L_{g}\left\|x^{r}\right\|_{\mathcal{B} \mathcal{P} \mathcal{C}_{h}^{0}}+\frac{M}{h\left(t^{r}\right)}\|g(0, \varphi)\|_{\mathcal{B}} \\
& +\frac{M}{h\left(t^{r}\right)} \int_{0}^{t^{r}} m(s) W(\mathcal{K} r+\mathcal{K} r h(s)+r h(s)) d s \\
& +M r \sum_{i=1}^{\infty} L_{i}+\frac{M}{h\left(t^{r}\right)} \sum_{i=1}^{\infty}\left\|I_{i}(0)\right\| \\
\leq L_{g}(1 & +M H) r+(1+M)\|g(0, \varphi)\|_{\mathcal{B}}+M \int_{0}^{t^{r}} \frac{m(s)((2 \mathcal{K}+1) r h(s))}{h(s)} d s
\end{aligned}
$$

and then,

$$
1 \leq L_{g}(1+M H)+M \lim _{r \rightarrow \infty} \inf \int_{0}^{+\infty} \frac{m(s)((2 \mathcal{K}+1) r h(s))}{r h(s)} d s+M \sum_{i=1}^{\infty} L_{i},
$$

which is contrary to $(3.10)$.

Let $r>0$ such that $\Gamma\left(B_{r}\right) \subset B_{r}$. From the proof of the Theorem 3.2, we know that $\Gamma_{2}$ is a completely continuous on $B_{r}$. Moreover, from the estimate,

$$
\begin{aligned}
\left\|\Gamma_{1} u-\Gamma_{1} v\right\|_{\mathcal{B P C}_{h}^{0}} & \leq L_{g}\|u-v\|_{\mathcal{B P C}_{h}^{0}}+H\|g(u, \varphi)-g(v, \varphi)\|_{\mathcal{B}} \\
& \leq L_{g}(1+H)\|u-v\|_{\mathcal{B P C}_{h}^{0}},
\end{aligned}
$$

we infer that $\Gamma_{1}$ is a contraction on $B_{r}$. It is easy to see that

$$
\left\|\Gamma_{3} u-\Gamma_{3} v\right\|_{\mathcal{B P C}_{h}^{0}} \leq M \sum_{i=1}^{\infty} L_{i}\|u-v\|_{\mathcal{B P C}_{h}^{0}}, u, v \in \mathcal{B P}_{\mathcal{P}} \mathcal{C}_{h}^{0},
$$

which implies that $\Gamma_{3}$ is a contraction in $\mathcal{B P C}_{h}^{0}(X)$ from which we conclude that $\Gamma$ is a condensing operator on $B_{r}$.

The assertion is now a consequence of Theorem 2.6.

\section{An APPLICATION}

To complete this work, we study the existence of global solutions for a concrete partial differential equation with nonlocal conditions. In the sequel, $X=L^{2}[0, \pi]$ and $A: D(A) \subset X \rightarrow X$ is the operator $A x=x^{\prime \prime}$ with domain $D(A)=\{x \in$ $\left.X: x^{\prime \prime} \in X, x(0)=x(\pi)=0\right\}$. It is well known that $A$ is the infinitesimal generator of an analytic semigroup $(T(t))_{t>0}$ on $X$. Furthermore, $A$ has discrete spectrum with eigenvalues of the form $-n^{2}, n \in \mathbb{N}$, and corresponding normalized 
eigenfunctions given by $z_{n}(x)=\left(\frac{2}{\pi}\right)^{\frac{1}{2}} \sin (n x)$; the set of functions $\left\{z_{n}: n \in \mathbb{N}\right\}$ is an orthonormal basis for $X$ and $T(t) x=\sum_{n=1}^{\infty} e^{-n^{2} t}\left\langle x, z_{n}\right\rangle z_{n}$ for every $x \in X$ and all $t \geq 0$. It follows from this last expression that $(T(t))_{t \geq 0}$ is a compact semigroup on $X$ and that $\|T(t)\| \leq e^{-t}$ for every $t \geq 0$.

As phase space, we choose the space $\mathcal{B}=\mathcal{P C}_{0} \times L^{2}(\rho, X)$, see Example 2.3, and we assume that the conditions (g5) and (g7) in [27] are verified. Under these conditions, $\mathcal{B}$ is a fading memory space, and as consequence, there exists $\mathcal{K}>0$ such that $\sup _{t>0}\{M(t), K(t)\} \leq \mathcal{K}$.

Consider the delayed impulsive partial differential equation with nonlocal conditions

$$
\begin{aligned}
\frac{\partial}{\partial t} w(t, \xi) & =\frac{\partial^{2}}{\partial \xi^{2}} w(t, \xi)+\int_{-\infty}^{t} a(t, s-t) w(s, \xi) d s+b(t, w(\rho(t), \xi)) \\
w(t, 0) & =w(t, \pi)=0, t \geq 0 \\
w(s, \xi) & =\sum_{i=1}^{\infty} L_{i} w\left(t_{i}+s, \xi\right)+\varphi(s, \xi), \quad s \leq 0, \xi \in[0, \pi] \\
\Delta w\left(t_{i}, \cdot\right) & =w\left(t_{i}^{+}, \cdot\right)-w\left(\cdot, t_{i}^{-}\right)=\int_{0}^{\pi} p_{i}\left(\xi, w\left(t_{i}, s\right)\right) d s
\end{aligned}
$$

for $t \geq 0$ and $\xi \in[0, \pi]$, and where $\left(L_{i}\right)_{i \in \mathbb{N}},\left(t_{i}\right)_{i \in \mathbb{N}}$ are sequences of real numbers and $\varphi \in \mathcal{B}$.

To treat this system in the abstract form (1.1)-(1.3), we assume the following conditions:

(a) Assume $\sum_{i=1}^{\infty}\left|L_{i}\right| h\left(t_{i}\right)<\infty$, the functions $\rho:[0, \infty) \rightarrow[0, \infty), a: \mathbb{R}^{2} \rightarrow \mathbb{R}$ are continuous and $\rho(t) \leq t$ and $L_{1}(t)=\left(\int_{-\infty}^{0} \frac{a^{2}(t, s)}{\rho(s)} d s\right)^{\frac{1}{2}}<\infty$, for every $t \geq 0$.

(b) Assume $b:[0, \infty) \times \mathbb{R} \rightarrow \mathbb{R}$ is continuous and that there exists a continuous function $L_{2}:[0, \infty) \rightarrow[0, \infty)$ such that $|b(t, x)| \leq L_{2}(t)|x|$ for every $t \geq 0$ and $x \in \mathbb{R}$.

(c) The functions $g: \mathcal{B P C}_{h}^{0}(X) \rightarrow \mathcal{B}, f:[0, \infty) \times \mathcal{B} \times X \rightarrow X$ by $g(u, \varphi)=$ $\varphi+\sum_{i=1}^{\infty} L_{i} u_{t_{i}}$ and

$$
f(t, \psi, x)(\xi)=\int_{-\infty}^{0} a(t, s) \psi(s, \xi) d s+b(t, x(\rho(t), \xi)) .
$$

(d) The functions $p_{i}:[0, \pi] \times \mathbb{R} \rightarrow \mathbb{R}, \quad i \in \mathbb{N}$, are continuous and there are positive constants $L_{i}$ such that

$$
\left|p_{i}(\xi, s)-p_{i}(\xi, \bar{s})\right| \leq L_{i}|s-\bar{s}|, \xi \in[0, \pi], s, \bar{s} \in \mathbb{R} .
$$

By defining the operator $I_{i}: X \rightarrow X$ by $I_{i}(x)(\xi)=\int_{0}^{\pi} p_{i}(\xi, x(0, s)) d s, i \in \mathbb{N}$, $\xi \in[0, \pi]$, we can transform system (4.1)-(4.4) into the abstract system (1.1)-(1.3). Moreover, it is easy to see that $f, g$ are continuous,

$$
\|f(t, \psi, x)\| \leq L_{1}(t)\|\psi\|_{\mathcal{B}}+L_{2}(t)\|x\|, \text { for all }(t, \psi, x) \in[0, \infty)
$$

and $g$ verifies conditions $\mathbf{H}_{\mathbf{3}}$ with $L_{g}: \mathcal{K} \sum_{i=1}^{\infty} L_{i} h\left(t_{i}\right)$.

The next result is a direct consequence from Theorem 3.3. We omit the proof. 
Proposition 4.1. If $\mathcal{K} \sum_{i=1}^{\infty} L_{i} h\left(t_{i}\right)+(1+2 \mathcal{K}) \int_{0}^{\infty}\left(L_{1}(s)+L_{2}(s)\right) d s$

$+\mathcal{K} \sum_{i=1}^{\infty} L_{i}<1$, then there exists a mild solution of (4.1)-(4.4).

\section{REFERENCES}

[1] H. Akca, A. Boucherif and V. Covachev, Impulsive functional differential equations with nonlocal conditions, Inter. J. Math. Math. Sci., 29:5 (2002), 251-256. 1

[2] A. Anguraj and K. Karthikeyan, Existence of solutions for impulsive neutral functional differential equations with nonlocal conditions, Nonlinear Anal., 70(7) (2009), 27172721. 1

[3] A. Anguraj and M. Mallika Arjunan, Existence and uniqueness of mild and classical solutions of impulsive evolution equations, Electronic Journal of Differential Equations, 111 (2005), 1-8. 1

[4] A. Anguraj and M. Mallika Arjunan, Existence results for an impulsive neutral integrodifferential equations in Banach spaces, Nonlinear Studies, 16(1) (2009), 33-48. 1

[5] D.D. Bainov and P.S. Simeonov, Impulsive Differential Equations: Periodic Solutions and Applications, Longman Scientific and Technical Group, England, 1993. 1

[6] K. Balachandran, J. Y. Park and M. Chandrasekaran, Nonlocal Cauchy problem for delay integrodifferential equations of Sobolve type in Banach spaces, Appl. Math. Lett., 15(7) (2002), 845-854. 1

[7] M. Benchohra, J. Henderson and S.K. Ntouyas, Existence results for impulsive multivalued semilinear neutral functional differential inclusions in Banach spaces, J. Math. Anal. Appl., 263(2) (2001), 763-780. 1

[8] M. Benchohra, J. Henderson and S.K. Ntouyas, Impulsive neutral functional differential inclusions in Banach spaces, Appl. Math. Lett., 15(8) (2002), 917-924. 1

[9] M. Benchohra and A. Ouahab, Impulsive neutral functional differential equations with variable times, Nonlinear Anal., 55(6) (2003), 679-693. 1

[10] M. Benchohra, J. Henderson and S.K. Ntouyas, Impulsive Differential Equations and Inclusions, Hindawi Publishing Corporation, New York, 2006. 1

[11] L. Byszewski, Theorems about the existence and uniqueness of solutions of a semilinear evolution nonlocal Cauchy problem, J. Math. Anal. Appl., 162(2) (1991), 494-505. 1

[12] L. Byszewski, Existence, uniqueness and asymptotic stability of solutions of abstract nonlocal Cauchy problems, Dynam. Systems Appl., 5(4) (1996), 595-605. 1

[13] L. Byszewski and H. Akca, Existence of solutions of a semilinear functional differential evolution nonlocal problem. Nonlinear Anal., 34(1) (1998), 65-72. 1

[14] L. Byszewski and H. Akca, On a mild solution of a semilinear functional-differential evolution nonlocal problem, J. Appl. Math. Stochastic Anal., 10(3) (1997), 265-271. 1

[15] Y.K. Chang, A. Anguraj and M. Mallika Arjunan, Existence results for non-densely defined neutral impulsive differential inclusions with nonlocal conditions, J. Appl. Math. Comput., 28 (2008), 79-91. 1

[16] Y.K. Chang, A. Anguraj and M. Mallika Arjunan, Existence results for impulsive neutral functional differential equations with infinite delay, Nonlinear Anal.: Hybrid Systems, 2(1) (2008), 209-218. 1

[17] Y.K. Chang, V. Kavitha and M. Mallika Arjunan, Existence results for impulsive neutral differential and integrodifferential equations with nonlocal conditions via fractional operators, Nonlinear Anal.: Hybrid Systems, 4(1) (2010), 32-43. 1

[18] C. Cuevas, E. Hernández and M. Rabelo, The existence of solutions for impulsive neutral functional differential equations, Comput. Math. Appl., 58 (2009), 744-757. 1

[19] A. Granas and J. Dugundji, Fixed Point Theory, Springer-Verlag, New York (2003). 2.5 
[20] E. Hernández, Existence results for partial neutral integrodifferential equations with nonlocal conditions, Dynam. Systems Appl., 11(2) (2002), 241-252. 1

[21] E. Hernández and S. M. Tanaka Aki, Global solutions for abstract functional differential equations with nonlocal conditions, Electronic Journal of Qualitative Theory of Differential Equations, 50 (2009), 1-8. 1

[22] E. Hernández, M. McKibben and Henríquez, H., Existence results for abstract impulsive second order neutral functional differential equations, Nonlinear Anal.,70 (2009), 2736-2751. 1

[23] E. Hernández, M. Pierri and G. Goncalves, Existence results for an impulsive abstract partial differential equations with state-dependent delay, Comput. Math. Appl. 52 (2006), 411-420. 1

[24] E. Hernández and M. Mckibben, Some comments on: "Existence of solutions of abstarct nonlinear second-order neutral functional integrodifferential equations" Comput. Math. Appl. 50(5-6) (2005), 655-669. 3

[25] E. Hernández, M. Rabello and H.R. Henriquez, Existence of solutions for impulsive partial neutral functional differential equations, J. Math. Anal. Appl., 331 (2007), 1135-1158. 1

[26] E. Hernández and H.R. Henriquez, Impulsive partial neutral differential equations, Appl. Math. Lett., 19 (2006), 215-222. 1

[27] Y. Hino, S. Murakami and T. Naito, In Functional-differential equations with infinite delay, Lecture notes in Mathematics, 1473, Springer-Verlog, Berlin (1991). 2.2, 2.3, 2, 4

[28] G. L. Karakostas and P. Ch. Tsamatos, Sufficient conditions for the existence of nonnegative solutions of a nonlocal boundary value problem, Appl. Math. Lett., 15(4) (2002), 401-407. 1

[29] V. Lakshmikantham, D.D. Bainov and P.S. Simeonov, Theory of Impulsive Differential Equations, World Scientific, Singapore, 1989. 1

[30] J.H. Liu, Nonlinear impulsive evolution equations, Dynam. Contin. Discrete Impuls. Sys., 6 (1999), 77-85. 1

[31] J. Liang, J.H. Liu and Ti-Jun Xiao, Nonlocal impulsive problems for nonlinear differential equations in Banach spaces, Math. Comput. Model., 49 (2009), 798-804. 1

[32] R. H. Martin, Nonlinear Operators and Differential Equations in Banach Spaces, Robert E. Krieger Publ. Co., Florida (1987). 2.6

[33] S. K. Ntouyas and P. Tsamatos, Global existence for semilinear evolution equations with nonlocal conditions, J. Math. Anal. Appl., 210 (1997), 679-687. 1

[34] W. E. Olmstead and C. A. Roberts, The one-dimensional heat equation with a nonlocal initial condition, Appl. Math. Lett., 10(3) (1997), 84-94. 1

[35] J.Y. Park, K. Balachandran and N. Annapoorani, Existence results for impulsive neutral functional integrodifferential equations with infinite delay, Nonlinear Analysis, 71 (2009), 3152-3162. 1

[36] A. Pazy, Semigroups of linear operators and applications to partial differential equations, Applied Mathematical Sciences, 44, Springer-Verlag, New York-Berlin (1983). 2

[37] Y.V. Rogovchenko, Impulsive evolution systems: Main results and new trends, $D y$ nam. Contin. Discrete Impuls. Syst., 3(1) (1997), 57-88. 1

[38] Y.V. Rogovchenko, Nonlinear impulsive evolution systems and application to population models, J. Math. Anal. Appl., 207(2) (1997), 300-315. 1

[39] A.M. Samoilenko and N.A Perestyuk, Impulsive Differential Equations, World Scientific, Singapore, 1995. 1

\footnotetext{
${ }^{1}$ Department of Mathematics, University College, Sungkyunkwan University, Suwon 440-746, South Korea

E-mail address: sdsiva@gmail.com
} 
${ }^{2}$ Department of Mathematics, Karunya University, Karunya Nagar, CoimbatORE - 641 114, TAMIL NADU, INDIA

E-mail address: arjunphd07@yahoo.co.in

${ }^{3}$ Department of Mathematics, Info Institute of Engineering, Kovilpalayam, Coimbatore - 641 107, TAmil Nadu, India

E-mail address: vijaysarovel@gmail.com 